\title{
Three-dimensional conformal geometry and prepotentials for four-dimensional fermionic higher-spin fields
}

\author{
Marc Henneaux, ${ }^{a, 1}$ Victor Lekeu, ${ }^{a, b}$ Amaury Leonard, ${ }^{a, c}$ Javier Matulich ${ }^{a}$ \\ and Stefan Prohazka ${ }^{a}$ \\ ${ }^{a}$ Université Libre de Bruxelles and International Solvay Institutes, \\ Campus Plaine - CP 231, B-1050 Bruxelles, Belgium \\ ${ }^{b}$ The Blackett Laboratory, Imperial College London, \\ Prince Consort Road London SWY 2AZ, U.K. \\ ${ }^{c}$ Max-Planck-Institut für Gravitationsphysik (Albert-Einstein-Institut), \\ Am Mühlenberg 1, DE-14476 Potsdam, Germany \\ E-mail: henneaux@ulb.ac.be, vlekeu@ulb.ac.be, \\ amaury.leonard@ulb.ac.be, jmatulic@ulb.ac.be, \\ stefan.prohazka@ulb.ac.be
}

ABSTRACT: We introduce prepotentials for fermionic higher-spin gauge fields in four spacetime dimensions, generalizing earlier work on bosonic fields. To that end, we first develop tools for handling conformal fermionic higher-spin gauge fields in three dimensions. This is necessary because the prepotentials turn out to be three-dimensional fields that enjoy both "higher-spin diffeomorphism" and "higher-spin Weyl" gauge symmetries. We discuss a number of the key properties of the relevant Cotton tensors. The reformulation of the equations of motion as "twisted self-duality conditions" is then exhibited. We show next how the Hamiltonian constraints can be explicitly solved in terms of appropriate prepotentials and show that the action takes then the same remarkable form for all spins.

Keywords: Higher Spin Symmetry, Gauge Symmetry, Supersymmetry and Duality

ARXiv EPrint: 1810.04457

\footnotetext{
${ }^{1}$ On leave of absence from Collège de France, Paris.
} 


\section{Contents}

1 Introduction 1

2 Three-dimensional conformal geometry 4

2.1 Riemann tensor $\quad 4$

2.2 Einstein tensor $\quad 5$

2.3 Schouten and Cotton tensors: first cases 5

$\begin{array}{lll}2.3 .1 & \text { Spin- } \frac{3}{2} & 6\end{array}$

2.3.2 Spin- $\frac{5}{2} \quad 7$

2.4 Schouten and Cotton tensors: general half-integer spin 11

$\begin{array}{lll}2.4 .1 & \text { Gauge completeness } & 13\end{array}$

$\begin{array}{lll}\text { 2.4.2 Conformal Poincaré lemma } & 14\end{array}$

3 Equations of motion as twisted self-duality 14

$\begin{array}{lll}3.1 & \text { From the Fronsdal to the Riemann tensor } & 15\end{array}$

$\begin{array}{lll}3.2 & \text { Rewriting as a twisted self-duality condition } & 16\end{array}$

$\begin{array}{lll}3.3 & \text { Hamiltonian constraint } & 17\end{array}$

4 Prepotentials $\quad 18$

$\begin{array}{ll}4.1 \text { Solution of the constraints } & 18\end{array}$

$\begin{array}{ll}4.2 & \text { Equations of motion } \\ & 19\end{array}$

$\begin{array}{lll}4.3 & \text { Action in terms of prepotentials } & 19\end{array}$

5 Comments and conclusions $\quad 21$

A Proof of $\gamma q=p \quad 22$

\section{Introduction}

Our paper is devoted to fermionic higher-spin conformal geometry in three dimensions and its application to the study of the dynamics of fermionic higher-spin gauge fields in four spacetime dimensions. More precisely, we consider massless fermionic higher-spin gauge fields of spin $s+\frac{1}{2}$, described by tensor-spinors $\psi_{i_{1} \cdots i_{s}}$ that are totally symmetric in their $s$ indices $i_{1}, \cdots, i_{s}$. Under (linearized) higher-spin diffeomorphisms and (linearized) higherspin Weyl transformations, these fields transform as

$$
\Gamma \psi_{i_{1} i_{2} \cdots i_{s}}=s \partial_{\left(i_{1}\right.} \xi_{\left.i_{2} \cdots i_{s}\right)}+s \gamma_{\left(i_{1}\right.} \lambda_{\left.i_{2} \cdots i_{s}\right)},
$$

where $\xi_{i_{1} \cdots i_{s-1}}$ and $\lambda_{i_{1} \cdots i_{s-1}}$ are symmetric tensor-spinors with $s-1$ indices. The first part of this transformation is also called a generalized diffeomorphism and the second one a 
generalized conformal transformation. The bosonic analogs of these transformations for a spin- $s$ field $Z_{i_{1} i_{2} \cdots i_{s}}$ are

$$
\Gamma Z_{i_{1} i_{2} \cdots i_{s}}=s \partial_{\left(i_{1}\right.} \xi_{\left.i_{2} \cdots i_{s}\right)}+\frac{s(s-1)}{2} \delta_{\left(i_{1} i_{2}\right.} \lambda_{\left.i_{3} \cdots i_{s}\right)},
$$

where $\xi_{i_{1} \cdots i_{s-1}}$ and $\lambda_{i_{1} \cdots i_{s-2}}$ are now symmetric tensors with $s-1$ indices and $s-2$ indices (and no spinor index), respectively. We have assumed for definiteness that the metric is Euclidean and given by $\delta_{i j}$, as this is the case relevant below. In the Minkowskian case, one simply needs to replace $\delta_{i j}$ by the Lorentzian metric $\eta_{i j}$ in (1.2). A central question investigated here is the construction of the invariants under both higher-spin diffeomorphisms and higher-spin Weyl transformations and the study of their properties. This is what we mean by "developing conformal geometry".

Conformal higher-spin gauge fields have attracted a lot of attention over the years, in any number of spacetime dimensions (for more information, see for instance some of the earliest references [1-10] and references therein). Conformal higher-spin gauge fields are interesting per se, but also appear as "prepotentials" in manifestly duality-invariant formulations of higher-spin non-conformal gauge fields in 4 spacetime dimensions, through the resolution of the constraints appearing in the Hamiltonian formalism. ${ }^{1}$ Following the work of [11], this was originally observed for spin-2 in [12-14] and generalized to higher integer spins in $[15,16]$. The prepotentials appear as three-dimensional tensors defined on the constant time Euclidean hypersurfaces in the $3+1$ Hamiltonian spacetime split. That they enjoy the higher-spin conformal gauge symmetry (1.2) was somehow unexpected but established for all integer spins $[15,16]$ using the relevant higher-spin conformal techniques in three dimensions.

For fermionic fields, however, prepotentials with the desired properties were introduced only for spins $\frac{3}{2}[17]$ and $\frac{5}{2}$ [18], where they were also verified to enjoy the symmetries (1.1). It was conjectured in that latter reference that a similar pattern would also hold for halfinteger spins equal to $\frac{7}{2}$ or higher, but that conjecture was not proven.

One reason that the conjecture was left unproved in [18] is that the corresponding tools for handling the higher-spin conformal symmetry in the dimension three relevant for the construction of prepotentials were not available in a form adapted to the Hamiltonian constraint analysis. The difficulty with dimension three is that conformal symmetry is not controlled by the Weyl tensor, which identically vanishes, but by the Cotton tensor, which involves higher derivatives of the fields.

The Cotton tensor has been defined for higher-spin bosonic gauge fields in [2, 19], and $[15]^{2}$ and its properties relevant to the introduction of prepotentials through the resolution of the Hamiltonian constraints have been established in [15] (see also [20]). The Cotton tensor contains $s-1$ derivatives of the Riemann tensor and thus $2 s-1$ derivatives of the higher-spin $s$ bosonic field. It plays a central role both in the study of the dynamics

\footnotetext{
${ }^{1}$ The term "prepotential" is always used here in that sense, as potentials needed to solve the constraints of the Hamiltonian formalism.

${ }^{2}$ Note a small subtlety between [2] and [15] when $s \geq 4$. It is that the definition given in [2] involves a symmetrization by hand, which turns out not to be necessary because the relevant expression is actually symmetric. This observation turns out to be useful for establishing the properties of the Cotton tensor.
} 
of conformal higher-spin gauge fields in three spacetime dimensions [21-25] and, as we have just pointed out, for the introduction of prepotentials in the Hamiltonian formulation of standard higher-spin gauge fields in four spacetime dimensions.

The purpose of this article is to extend the work of $[15,16]$ to higher-spin fermionic fields. To that end, we define and study the properties of the Cotton tensor for half-integer spin fields in three dimensions. The Cotton tensor is actually a tensor-spinor, but like for any other tensor-spinor we shall often loosely refer to it just as tensor. The Cotton tensor contains $2 s$ derivatives of the field $\psi_{i_{1} i_{2} \cdots i_{s}}$ (in terms of the spin $S=s+\frac{1}{2}$, this is equal to $2 S-1$ as in the bosonic case). It was defined earlier in [26] for spin $\frac{3}{2}$ and more recently for all half-integer spins in [27-29]. Our derivation follows a different line. It is based on the use of the differential operator $d_{(s)}$ of $[30,31]$ that fulfills

$$
d_{(s)}^{s+1}=0
$$

and the corresponding Poincaré-type lemmas. As such, our definition it is not tied to supersymmetry or superspace calculus. The same method has been applied to mixed Young symmetry tensors for which the "critical dimension" where the Weyl tensor identically vanishes is generically higher than 3 (see, e.g., [32] and [33-36]).

Once the Cotton tensor has been defined and its main properties established, one can turn to the resolution of the fermionic constraints of the Hamiltonian formalism. These can be rewritten in a form that makes the introduction of prepotentials effortless.

Our paper is organized as follows. Section 2 is devoted to the definition and study of the properties of the Cotton tensor for half-integer spin fields in three dimensions. We then consider in section 3 the dynamics. We first show that the equations of motion can be rewritten, just as in the bosonic case [16], as twisted self-duality conditions ([37-39]). We then turn to the Hamiltonian formulation of the equations of motion, in particular to the constraint equation, which plays a central role in the twisted self-duality conditions. We solve in section 4 the constraints, which is the step that introduces the prepotentials in terms of which we rewrite the action. This action enjoys a chiral SO(2) symmetry. Section 5 is devoted to final comments and conclusions. Table 1, appended at the end of this work, summarizes the most important definitions and properties of bosonic and fermionic higher spin fields in the prepotential formalism and might be useful to get a fast overview.

Notation and conventions. The flat metric of 4-dimensional spacetime has signature $(-,+,+,+)$ and its spatial sections are Euclidean with signature $(+,+,+)$. Our convention for the Dirac $\gamma$ matrices is that $\left\{\gamma_{\mu}, \gamma_{\nu}\right\}=2 \eta_{\mu \nu}$ where $\eta_{\mu \nu}$ is the spacetime metric. Furthermore, we define $\gamma_{5} \equiv \gamma_{0} \gamma_{1} \gamma_{2} \gamma_{3}$, so that the spatial gamma matrices satisfy the useful identity

$$
\gamma_{i} \gamma_{j}=\delta_{i j}+\varepsilon_{i j k} \gamma^{k} \gamma_{5} \gamma_{0}
$$

and $\gamma_{i j} \equiv \gamma_{[i} \gamma_{j]}=\varepsilon_{i j k} \gamma^{k} \gamma_{5} \gamma_{0}$ with $\varepsilon_{123}=\varepsilon^{123}=1$. Notice that $\left(\gamma_{5}\right)^{2}=-I$.

Taking one spatial trace is indicated with a bar, $\bar{T}=T^{[1]}=\delta^{i j} T_{i j}$, and the slash is the spatial gamma-trace, $T=\gamma^{i} T_{i}$. Multiple traces are indicated by a bracketed exponent, e.g., $T^{[2]}=\delta^{i j} \delta^{k l} T_{i j k l}$. Due to the property $\gamma^{(i} \gamma^{j)}=\delta^{i j}$, the double gamma-trace of a 
symmetric tensor is the same as a normal trace, so no notation is introduced for multiple gamma-traces. Space-time traces are rarely used and indicated by a prime or backslash respectively, $T^{\prime}=\eta^{\mu \nu} T_{\mu \nu}$ and $\mathbb{T}=\gamma^{\mu} T_{\mu}$. The Dirac conjugate $\bar{\psi}=\psi^{\dagger} \gamma^{0}$ is also indicated by a bar, but no confusion should arise since the context is clear.

We will sometimes find it convenient to adopt a compact notation where the vectorial indices are suppressed and symmetrization over unwritten vectorial indices is implied. For example, in this notation, equations (1.1) and (1.2) become

$$
\Gamma \psi=s \partial \xi+s \gamma \lambda, \quad \Gamma Z=s \partial \xi+\frac{s(s-1)}{2} \delta \lambda .
$$

Since it should be clear from the context and to improve readability we mostly call tensorspinors just tensors.

Finally, a tensor(-spinor) with $\left(a_{1}, a_{2}, \cdots, a_{n}\right)$ Young symmetry is labeled by the length of the rows, i.e., corresponds to a Young diagram with $n$ rows which have $a_{i}$ boxes. If not stated otherwise, we follow the manifestly antisymmetric convention.

\section{Three-dimensional conformal geometry}

The Riemann tensor, or equivalently the Einstein tensor, controls higher-spin diffeomorphisms. By this we mean that any function that is higher-spin diffeomorphism invariant can be written as a function of the Riemann (or equivalently Einstein) tensor and its derivatives. However, the Riemann tensor lacks higher-spin conformal invariance, which is an important property needed for the resolution of the Hamiltonian constraints. For this reason the Cotton tensor must be introduced and its important properties established.

\subsection{Riemann tensor}

The "Riemann", or "curvature" tensor is defined by taking $s$ derivatives of the spin-s (or $s+\frac{1}{2}$ ) field [40]. In terms of the differential operator $d_{(s)}$ of $[30,31]$, the Riemann tensor can be written as

$$
R=d_{(s)}^{s} \psi
$$

or, in components,

$$
R_{i_{1} j_{1} \cdots i_{s} j_{s}}=2^{s} \partial_{\left[j_{1} \mid\right.} \cdots \partial_{\left[j_{s} \mid\right.} \psi_{\left.\left.\mid i_{1}\right] \cdots \mid i_{s}\right]} .
$$

It is a tensor of Young symmetry type $(s, s)$ which satisfies the Bianchi identity $d_{(s)} R=0$ because of the property $d_{(s)}^{s+1}=0$. On account of that same equation, it is also invariant under higher-spin diffeomorphisms, which can be written as $\Gamma_{\xi} \psi=d_{(s)} \xi$. Furthermore, a necessary and sufficient condition for the higher-spin field to be a pure higher-spin diffeomorphism, i.e., $\psi=d_{(s)} \xi$ for some $\xi$, is that its Riemann tensor vanishes. This is equivalent to the statement that the most general higher-spin diffeomorphism invariant function can be expressed as a function of the Riemann tensor and its derivatives only.

The Riemann tensor is not invariant under higher-spin Weyl transformations. The construction of invariants for that symmetry makes dimension three very special. In dimension strictly greater than three, one can construct invariants by removing gamma-trace 
terms from the Riemann tensor, defining thereby the Weyl tensor. This procedure does not yield quantities of great interest in dimension three, however, because the tracefree part of the Riemann tensor then identically vanishes. What controls higher-spin Weyl symmetry is the Cotton tensor, which contains higher derivatives of the higher-spin fields, to which we will turn after defining the Einstein tensor for general $s$.

\subsection{Einstein tensor}

As we indicated, the Weyl tensor identically vanishes in three dimensions. The curvature tensor is therefore completely determined by the "Ricci tensor", or equivalently, by the "Einstein tensor", which is the $s$ times dual (with a sign factor inserted for convenience)

$$
G=(-1)^{s} \underbrace{\star \star \cdots \star}_{s \text { times }} d_{(s)}^{s} \psi
$$

of the curvature. We dualize on each antisymmetric pair so this expression can also be written as

$$
G=(\varepsilon \cdot \partial \cdot)^{s} \psi
$$

In words, the Einstein tensor is obtained by contracting $s$ times $\varepsilon_{i j k_{r}} \partial^{j}$ with $\psi^{k_{1} \cdots k_{s}}$. Explicitly, when $s=1$ ( $\left.\operatorname{spin} \frac{3}{2}\right)$ one has

$$
G_{i}=\varepsilon_{i j k} \partial^{j} \psi^{k}
$$

while for $s=2\left(\operatorname{spin} \frac{5}{2}\right)$

$$
G_{i j}=\varepsilon_{i k m} \varepsilon_{j l n} \partial^{k} \partial^{l} \psi^{m n} .
$$

The Einstein tensor is a completely symmetric tensor which fulfills the contracted Bianchi identity

$$
\partial_{i_{1}} G^{i_{1} i_{2} \cdots i_{s}}=0 .
$$

Conversely, any symmetric and divergenceless tensor can be written as the Einstein tensor of some field.

While equivalent, we find it convenient in the sequel to work systematically with the Einstein tensor rather than with the Riemann tensor.

\subsection{Schouten and Cotton tensors: first cases}

The Einstein tensor and its derivatives provide a complete set of higher-spin diffeomorphism invariant functions, but little can be said about higher-spin Weyl symmetry without introducing the Cotton tensor. The idea is to algebraically construct out of the Einstein tensor and its successive traces the "Schouten tensor" that transforms under higher-spin Weyl transformations into a symmetrized gradient. The Cotton tensor is then the Einstein tensor of the Schouten tensor and is therefore Weyl invariant. This is much along the lines of the bosonic case, as can be seen in table 1 at the end of this work, but there the Schouten tensor transforms under a symmetrized double gradient rather than a single symmetrized gradient. The symmetrized double gradient is removed by acting with $d_{(s)}^{s-1}$ rather than 
with $d_{(s)}^{s}$, which explains the difference in the number of derivatives when expressed in terms of $s$.

After the Cotton tensor is properly defined, we prove the following two important theorems.

Gauge completeness: the Cotton tensor is zero if and only if the field is pure gauge with respect to higher-spin diffeomorphisms and higher-spin Weyl transformations.

This property ensures that the Cotton tensor fully controls the gauge invariance, which means that any local higher-spin diffeomorphism and higher-spin Weyl invariant function can be written in terms of the Cotton tensor and its derivatives. So the Cotton tensor and its derivatives provide a complete set of gauge invariant functions (see, e.g., appendix B.1 of [15] for more details and the proof that this claim is equivalent to the property above).

Conformal Poincaré lemma: any symmetric, divergenceless and gamma-traceless tensor can be written as the Cotton tensor of some field.

This property will be crucial when solving the Hamiltonian constraint of the higherspin fermionic field in section 4 .

As a warm-up, we start with the spin- $\frac{3}{2}$ field. Most of the subtleties of the general case are already present in the case of the spin- $\frac{5}{2}$ field, which we discuss next before we generalize to general spin.

\subsubsection{Spin- $\frac{3}{2}$}

We first consider the familiar case of $\operatorname{spin} \frac{3}{2}(s=1)$. The spin- $\frac{3}{2}$ field is a vector-spinor $\psi_{i}$. We are looking for a complete set of functions of this field invariant under the following transformations

$$
\Gamma \psi_{i}=\partial_{i} \xi+\gamma_{i} \lambda .
$$

As we have recalled, a complete set of invariants under spin- $\frac{3}{2}$ diffeomorphisms, i.e., the first term in (2.8), is given by the Einstein tensor

$$
G_{i}=\varepsilon_{i j k} \partial^{j} \psi^{k}
$$

and its derivatives, which transforms under a conformal transformation as $\Gamma G_{i}=\varepsilon_{i j k} \partial^{j} \gamma^{k} \lambda$. This implies $\Gamma \notin \mathbb{r}=-2 \gamma_{5} \gamma_{0} \not \partial \lambda$ and leads us to define a Schouten tensor as

$$
S_{i}=G_{i}-\frac{1}{2} \gamma_{i} \phi_{i}
$$

Its gauge variation is indeed a gradient

$$
\Gamma S_{i}=\partial_{i}\left(\gamma_{5} \gamma_{0} \lambda\right)
$$

The definition (2.10) is invertible: the Einstein tensor can be expressed in terms of the Schouten as

$$
G_{i}=S_{i}-\gamma_{i} \phi
$$


Since the Einstein tensor is identically divergenceless, the Schouten satisfies

$$
0=\partial^{i} S_{i}-\not \partial \$
$$

This is the Bianchi identity for the Schouten tensor. The conformal invariant is then the Einstein tensor of the Schouten tensor, which we name "Cotton tensor". For the spin- $\frac{3}{2}$ field this quantity is given by

$$
\begin{aligned}
D_{i} & =\varepsilon_{i j k} \partial^{j} S^{k} \\
& =\frac{1}{2}\left(\partial_{i} \partial^{j} \psi_{j}-\Delta \psi_{i}\right)-\frac{1}{2} \varepsilon_{i j k} \gamma_{5} \gamma_{0} \not \partial \partial^{j} \psi^{k} .
\end{aligned}
$$

It is identically divergenceless, and also gamma-traceless on account of (2.13) (and (1.4)). It is called "Cottino" in [26] where it was first introduced.

We will now prove that the Cotton tensor and its derivatives provide a complete set of invariant functions with respect to higher-spin diffeomorphism and Weyl transformations (gauge completeness), and any tensor that is both gamma-traceless and divergenceless is the Cotton tensor of some vector-spinor ("conformal Poincaré lemma").

Gauge completeness. The first property is equivalent to the fact that $D_{i}=0$ is a necessary and sufficient condition for the spin- $\frac{3}{2}$ field to be pure gauge. By construction, we have $\Gamma D_{i}=0$ which shows that for a pure gauge field the Cotton tensor necessarily vanishes. This condition is also sufficient, since if $D_{i}=\varepsilon_{i j k} \partial^{j} S^{k}=0$, then (using the Poincaré lemma with a spectator spinor index) we have $S_{i}=\partial_{i} \rho$ for some $\rho$ that we can always write as $\rho=\gamma_{5} \gamma_{0} \lambda$. Inserting now $S_{i}$ into (2.12) leads to $G_{i}-\varepsilon_{i j k} \partial^{j} \gamma^{k} \lambda=0$, or equivalently $\varepsilon_{i j k} \partial^{j}\left(\psi^{k}-\gamma^{k} \lambda\right)=0$. We can again use the Poincaré lemma, yielding $\psi_{i}=\partial_{i} \xi+\gamma_{i} \lambda$ for some $\xi$. Therefore a vanishing Cotton tensor also implies that the field is pure gauge.

Conformal Poincaré lemma. Furthermore, by running backwards the construction of the Cotton tensor, it is also easy to see that any vector-spinor field $T_{i}$ that is both gammatraceless and divergenceless, $\partial^{i} T_{i}=0$ and $T=0$, is the Cotton tensor of some vector-spinor field $\psi_{j}$, i.e., $T=D[\psi]$.

Indeed, the condition $\partial^{i} T_{i}=0$ implies $T_{i}=\varepsilon_{i j k} \partial^{j} S^{k}$ for some $S^{k}$ that fulfills $\partial^{i} S_{i}-$ $\not \phi \$=0$ on account of $T=0$. We can now define a tensor $G_{i}$ through $G_{i}=S_{i}-\gamma_{i} \phi$ : it fulfills $\partial^{i} G_{i}=0$ and is thus itself equal to $G_{i}=\varepsilon_{i j k} \partial^{j} \psi^{k}$ for some $\psi_{i}$ which is the searched-for vector-spinor.

\subsubsection{Spin- $\frac{5}{2}$}

We now turn to the discussion of the spin- $\frac{5}{2}$ field, a symmetric tensor(-spinor) $\psi_{i j}$, which will lay the ground work for the next section where general half-integer spin is considered. We are looking for a complete set of functions that is invariant under the transformations

$$
\Gamma \psi_{i j}=2 \partial_{(i} \xi_{j)}+2 \gamma_{(i} \lambda_{j)} .
$$


As we have seen, such a complete set of invariants under spin- $\frac{5}{2}$ diffeomorphisms is given by the Einstein tensor

$$
\begin{aligned}
G_{i j} & =\varepsilon_{i k m} \varepsilon_{j l n} \partial^{k} \partial^{l} \psi^{m n} \\
& =\delta_{i j}\left(\Delta \bar{\psi}-\partial^{k} \partial^{l} \psi_{k l}\right)+2 \partial_{(i} \partial^{k} \psi_{j) k}-\Delta \psi_{i j}-\partial_{i} \partial_{j} \bar{\psi}
\end{aligned}
$$

and its derivatives. The variation of this tensor under a Weyl transformation is given by

$$
\Gamma G_{i j}=2 \varepsilon_{(i \mid k m} \partial^{k} \gamma^{m} \mu_{\mid j)}
$$

where $\mu_{j}=\varepsilon_{j l n} \partial^{l} \lambda^{n}$ is the Einstein tensor of $\lambda^{n}$. For its traces, this implies

$$
\begin{aligned}
\Gamma \mathscr{G}_{i} & =-3 \gamma_{5} \gamma_{0} \not \partial \mu_{i}+\varepsilon_{i j k} \partial^{k} \mu^{m} \\
\Gamma \bar{G} & =\Gamma \delta^{i j} G_{i j}=2 \varepsilon_{i j k} \partial^{i} \gamma^{j} \mu^{k} .
\end{aligned}
$$

The Schouten tensor is a combination of the Einstein tensor and its traces, i.e., $S=$ $G+a_{1} \bar{G}+b_{0} \gamma G_{r}$. Using this ansatz and the condition that the Schouten tensor should vary to a symmetrized derivative leads to ${ }^{3}$

$$
S_{i j}=G_{i j}-\frac{1}{4} \delta_{i j} \bar{G}-\frac{1}{2} \gamma_{(i} \phi_{i j)}
$$

Indeed, it varies to

$$
\Gamma S_{i j}=\partial_{(i} \nu_{j}
$$

where we have defined

$$
\nu_{i}=-\frac{1}{2} \varepsilon_{i j k} \gamma^{j} \mu^{k}+\frac{3}{2} \gamma_{5} \gamma_{0} \mu_{i}
$$

As in the spin- $\frac{3}{2}$ case, the relation between the Einstein and Schouten tensors is invertible and we have

$$
G_{i j}=S_{i j}-2 \gamma_{(i} \phi_{j)}-\delta_{i j} \bar{S}
$$

which implies $\mathbb{G}_{i}=-4 \$_{i}$ and $\bar{G}=-4 \bar{S}$ for the traces. Since the Einstein tensor is identically divergenceless, the Schouten tensor satisfies $0=\partial^{j} S_{i j}-\not \partial \$_{i}-\gamma_{i} \partial_{j} \not^{j}-\partial_{i} \bar{S}$. Taking the gamma-trace of this expression gives $\partial_{i} \phi^{i}=0$, which then implies the Bianchi identity for the Schouten tensor

$$
U_{i}[S] \equiv \partial^{j} S_{i j}-\not \partial \$_{i}-\partial_{i} \bar{S}=0
$$

which is equivalent to the divergencelessness of the Einstein tensor. Likewise, the relation between $\mu_{i}$ and $\nu_{i}$ can be inverted to

$$
\mu^{i}=-\frac{1}{2} \varepsilon^{i j k} \gamma_{j} \nu_{k}-\gamma_{5} \gamma_{0} \nu_{i}
$$

\footnotetext{
${ }^{3}$ Note that the Schouten tensor used in [18] is the Schouten tensor for a spin-2 field with spinor indices treated as spectator indices. The definition adopted here, which is different, is more adapted to the spin- $\frac{5}{2}$ case. The tensor (2.22) enjoys indeed more useful properties.
} 
and the property $\partial_{i} \mu^{i}=0$ is equivalent to the identity $\partial_{i} \nu^{i}-\frac{1}{2} \gamma_{5} \gamma_{0} \varepsilon^{i j k} \partial^{i} \gamma_{j} \nu_{k}=0$ satisfied by $\nu_{i}$, which can also be rewritten as

$$
I[\nu] \equiv \partial_{i} \nu^{i}+\not \partial \psi=0 .
$$

An important property is that

$$
U_{i}[\partial \nu]=-\frac{1}{2} \partial_{i} I[\nu]
$$

which shows that the Bianchi identity for the Schouten tensor is compatible with Weyl transformations.

The conformal invariant is then the Einstein tensor of the Schouten tensor, named the "Cotton tensor"

$$
\begin{aligned}
D_{i j} & =\varepsilon_{i k m} \varepsilon_{j l n} \partial^{k} \partial^{l} S^{m n} \\
& =2 \partial_{(i} \partial^{k} S_{j) k}-\Delta S_{i j}-\partial_{i} \partial_{j} \bar{S}
\end{aligned}
$$

It is again divergenceless and gamma-traceless, and invariant under higher-spin diffeomorphisms and Weyl transformations. Its explicit form in terms of the fourth derivatives of $\psi_{i j}$ is

$$
\begin{aligned}
D_{i j}= & \Delta^{2}\left(\psi_{i j}-\frac{1}{2} \gamma_{(i} \psi_{j)}-\frac{1}{4} \delta_{i j} \bar{\psi}\right) \\
& +\frac{\Delta}{4}\left(\partial_{i} \partial_{j} \bar{\psi}+2 \not \partial \partial_{(i} \psi_{j)}+\partial^{k}\left(\delta_{i j} \partial^{l} \psi_{l k}-10 \partial_{(i} \psi_{j) k}+2 \gamma_{(i} \not \partial \psi_{j) k}+2 \partial_{(i} \gamma_{j)} \psi_{k}\right)\right) \\
& +\frac{1}{4} \partial_{i} \partial_{j}\left(5 \partial^{k} \partial^{l} \psi_{k l}-2 \not \partial \partial^{k} \psi_{k}\right)-\frac{1}{2} \partial_{(i} \gamma_{j)} \partial^{k} \partial^{l} \not \partial \psi_{k l},
\end{aligned}
$$

an expression that would have been of course very difficult to guess (and to generalize) without the systematic construction using Einstein and Schouten tensors.

We now turn to the proof of the two important theorems concerning the Cotton tensor.

Gauge completeness. We first want to show that the Cotton tensor fully characterizes the spin- $\frac{5}{2}$ diffeomorphism and Weyl-invariance. For that, we need to show that the condition $D_{i j}=0$ is equivalent to $\psi_{i j}$ being pure gauge.

If $\psi_{i j}$ is pure gauge, then the Cotton vanishes by construction, $D_{i j}=0$. Conversely, if $D_{i j}=0$, the Schouten satisfies $\partial_{[i} S_{j][k, l]}=0$ or, in index-free notation, $d_{(2)}^{2} S=0$. Using the Poincaré lemma for two-column Young tableaux [30,31], this implies that $S=d_{(2)} \nu$ for some vector $\nu$, i.e., $\left.S_{i j}=\partial_{(i} \nu_{j}\right)$.

Defining $G^{i j}$ and $\mu^{i}$ through $S_{i j}$ and $\nu_{i}$ by equations (2.25) and (2.27) gives $G_{i j}=$ $2 \varepsilon_{(i \mid a b} \partial^{a} \gamma^{b} \mu_{\mid j)}$. It is proven below that the ambiguity in $\nu_{i}$ allows us to fix $\partial_{i} \mu^{i}=0$. This implies $\mu^{i}=\varepsilon^{i j k} \partial_{j} \lambda_{k}$ for some $\lambda_{k}$ and $G_{i j}=\varepsilon_{i k m} \varepsilon_{j l n} \partial^{k} \partial^{l}\left(2 \gamma^{(m} \lambda^{n)}\right)$, or $\varepsilon_{i k m} \varepsilon_{j l n} \partial^{k} \partial^{l}\left(\psi^{m n}-\right.$ $\left.2 \gamma^{(m} \lambda^{n)}\right)=0$. Again using the relevant Poincaré lemma, this implies $\psi^{m n}=2 \partial^{(m} \xi^{n)}+$ $2 \gamma^{(m} \lambda^{n)}$ for some $\xi^{n}$, which shows that $\psi^{m n}$ is pure gauge.

The only extra step with respect to the spin $\frac{3}{2}$-case consists thus in establishing that the ambiguity in $\nu_{i}$ allows us to fix $\partial_{i} \mu^{i}=0$ or, equivalently, $I[\nu]=0$. Due to the Bianchi identity $U[S]=0$ satisfied by the Schouten tensor, we know that $I[\nu]$ satisfies $\partial_{i} I[\nu]=0$, 
which leads to $I[\nu]=p^{(0)}$ with constant $p^{(0)}$. We can also redefine $\nu_{i}$ as $\nu_{i} \sim \nu_{i}+\tilde{\nu}_{i}$ without changing the Schouten tensor, as long as $\tilde{\nu}_{i}$ satisfies $\partial_{(i} \tilde{\nu}_{j)}=0$. This is just the Killing equation for flat space (with a spectator spinor index) and is solved by $\tilde{\nu}_{i}=q_{i}^{(0)}+q_{i j}^{(1)} x^{j}$, where $q_{i}^{(0)}$ is a constant vector-spinor and $q_{i j}^{(1)}$ is antisymmetric. A short computation then shows that

$$
I[\nu+\tilde{\nu}]=p^{(0)}-\gamma^{i j} q_{i j}^{(1)} .
$$

Therefore, choosing $q_{i j}^{(1)}=-\frac{1}{6} \gamma_{i j} p$ fixes $I[\nu+\tilde{\nu}]=0$, thus concluding the proof.

Conformal Poincaré lemma. The Cotton tensor is symmetric, divergenceless and gamma-traceless. We will now prove that any tensor with these properties, i.e., any symmetric tensor $T_{i j}$ satisfying $\partial^{i} T_{i j}=0$ and $T_{i}=0$, can be written as the Cotton tensor $T_{i j}=D_{i j}[\psi]$ for some $\psi_{i j}$.

Divergenceless of a symmetric tensor implies, using the generalized Poincaré lemma [30, 31], that $T_{i j}$ is the Einstein tensor of some symmetric tensor $S_{i j}$,

$$
T_{i j}=\varepsilon_{i k m} \varepsilon_{j l n} \partial^{k} \partial^{l} S^{m n} .
$$

The condition $T_{i}=0$ leads to

$$
\begin{aligned}
0 & =\varepsilon_{i j k} \partial^{j}\left(\partial_{l} S^{l k}-\not \partial \phi^{k}-\partial^{k} \bar{S}\right) \\
& =\varepsilon_{i j k} \partial^{j} U^{k}[S],
\end{aligned}
$$

where we have added the last trace-term for convenience, at no cost since partial derivatives commute while $\varepsilon_{i j k}=-\varepsilon_{i k j}$. The Poincaré lemma then implies

$$
U_{i}[S]=\partial^{j} S_{i j}-\not \partial \$_{i}-\partial_{i} \bar{S}=\partial_{i} \rho
$$

Suppose for now that the right hand side vanishes. Then, our tensor $S$ satisfies the Bianchi identity (2.26) for the Schouten tensor of that spin. Therefore, the tensor $G_{i j}$ defined by

$$
G_{i j}=S_{i j}-2 \gamma_{(i} \phi_{j)}-\delta_{i j} \bar{S}
$$

satisfies $\partial^{i} G_{i j}=0$, as is proven in the beginning of this section. This followed from the invertibility of the definition of the Schouten in terms of the Einstein tensor, for which the definition in terms of $\psi$ was irrelevant. Now, since $G$ is divergenceless, we have

$$
G_{i j}=\varepsilon_{i k m} \varepsilon_{j l n} \partial^{k} \partial^{l} \psi^{m n}
$$

for some symmetric $\psi^{m n}$, which shows that $S$ is the Schouten tensor of $\psi$ and therefore that $T_{i j}$ is its Cotton, $T_{i j}=D_{i j}[\psi]$.

To finish the proof we need to show that we can use the ambiguities in the Poincaré lemma to indeed set the right hand side of (2.37) to zero. The freedom we have is given by $S_{i j} \sim S_{i j}+\partial_{(i} \nu_{j)}$ which leads to

$$
U_{i}[\partial \nu]=-\frac{1}{2} \partial_{i}\left(\partial_{j} \nu^{j}+\not \partial \psi\right)=\partial_{i} \rho
$$


Therefore, it is sufficient to choose $\nu$ such that

$$
I[\nu]=\partial_{i} \nu^{i}+\not \partial \psi=-2 \rho .
$$

To prove that such a $\nu_{i}$ exists, we define a vector-spinor $\mu^{i}$ in terms of $\nu^{i}$ as in equation (2.27) and use the invertibility of that relation. Equation (2.41) is then equivalent to

$$
\partial_{i} \mu^{i}=\hat{\rho}
$$

where $\hat{\rho}=\gamma_{5} \gamma_{0} \rho$, which always has a solution. This therefore completes the proof.

\subsection{Schouten and Cotton tensors: general half-integer spin}

The spin- $\left(s+\frac{1}{2}\right)$ field is a symmetric tensor(-spinor) with $s$ vectorial indices $\psi$. Again, a complete set of invariants under spin- $\left(s+\frac{1}{2}\right)$ diffeomorphisms is given by the Einstein tensor

$$
G=(\varepsilon \cdot \partial \cdot)^{s} \psi
$$

The variation of this tensor under a Weyl transformation (1.1) is

$$
\Gamma G=s(\varepsilon \cdot \partial \cdot \gamma) \mu,
$$

where $\mu=(\epsilon \cdot \partial \cdot)^{s-1} \lambda$ is the Einstein tensor of $\lambda$ or, equivalently, any symmetric divergenceless tensor of rank $s-1$. This implies, for the $p$-th trace and gamma-trace of $G$

$$
\begin{aligned}
& \Gamma G^{[p]}=2 p(\varepsilon \cdot \partial \cdot \gamma \cdot) \mu^{[p-1]}+(s-2 p)(\varepsilon \cdot \partial \cdot \gamma) \mu^{[p]} \quad(0 \leq p \leq\lfloor s / 2\rfloor), \\
& \Gamma G_{\tau}^{[p]}=-\gamma_{5} \gamma_{0}(s+1) \not \partial \mu^{[p]}+(s-1-2 p)(\varepsilon \cdot \partial \cdot) \mu^{[p]} \quad(0 \leq p \leq\lfloor(s-1) / 2\rfloor) \text {, }
\end{aligned}
$$

where $\lfloor s / 2\rfloor$ denotes the largest integer equal or smaller than $s / 2$. The Schouten tensor will be built out of these quantities multiplied by $p$ delta functions and an additional gamma matrix for $G_{c}^{[p]}$. The variation of these terms is given by

$$
\begin{aligned}
\delta^{p} \Gamma G^{[p]}= & 2 p \delta^{p}(\varepsilon \cdot \partial \cdot \gamma \cdot) \mu^{[p-1]}+(s-2 p) \delta^{p}(\varepsilon \cdot \partial \cdot \gamma) \mu^{[p]} \\
\delta^{p} \gamma \Gamma G^{[p]}= & -\gamma_{5} \gamma_{0}(s+1) \delta^{p} \partial \mu^{[p]}+(s+1) \delta^{p}(\varepsilon \cdot \partial \cdot \gamma) \mu^{[p]} \\
& +(s-1-2 p) \delta^{p} \gamma(\varepsilon \cdot \partial \cdot) \mu^{[p]}
\end{aligned}
$$

At first sight, it does not seem possible to combine these expressions in order to obtain a symmetrized derivative, but we have to take into account the following identity

$$
\begin{aligned}
0 & =4 \varepsilon_{[i j k} \delta_{l] m} \partial^{i} \gamma^{j} \mu^{k} \\
& =\varepsilon_{i j k} \delta_{l m} \partial^{i} \gamma^{j} \mu^{k}-\varepsilon_{j k l} \partial_{m} \gamma^{j} \mu^{k}+\varepsilon_{k l i} \partial^{i} \gamma_{m} \mu^{k}-\varepsilon_{l i j} \partial^{i} \gamma^{j} \mu^{m},
\end{aligned}
$$

where the spectator indices of $\mu$ have been left unwritten. After symmetrization in $\mathrm{lm}$ (together with the remaining indices of $\mu$ ), this gives

$$
0=\delta \varepsilon \cdot \partial \cdot \gamma \cdot \mu-\partial(\varepsilon \cdot \gamma \cdot) \mu+\gamma(\varepsilon \cdot \partial \cdot) \mu-(\varepsilon \cdot \partial \cdot \gamma) \mu
$$


which in turn implies

$$
\begin{aligned}
\delta^{p} \Gamma G^{[p]}= & 2 p \delta^{p-1} \partial(\varepsilon \cdot \gamma \cdot) \mu^{[p-1]}-2 p \delta^{p-1} \gamma(\varepsilon \cdot \partial \cdot) \mu^{[p-1]}+2 p \delta^{p-1}(\varepsilon \cdot \partial \cdot \gamma) \mu^{[p-1]} \\
& +(s-2 p) \delta^{p}(\varepsilon \cdot \partial \cdot \gamma) \mu^{[p]} .
\end{aligned}
$$

This leads us to define the Schouten tensor as

$$
S=\sum_{p=0}^{\lfloor s / 2\rfloor} a_{p} \delta^{p} G^{[p]}+\sum_{p=0}^{\lfloor(s-1) / 2\rfloor} b_{p} \delta^{p} \gamma \phi_{i}^{[p]} .
$$

Requiring the gauge variation of the Schouten to be a symmetrized derivative $(\Gamma S=\partial \nu$ for some symmetric tensor $\nu$ ) imposes

$$
\begin{aligned}
& 0=-2(p+1) a_{p+1}+(s-1-2 p) b_{p}, \\
& 0=2(p+1) a_{p+1}+(s-2 p) a_{p}+(s+1) b_{p} .
\end{aligned}
$$

Taking the initial condition $a_{0}=1$, the solution to these recurrence relations is

$$
\begin{aligned}
a_{p} & =\frac{(-1)^{p}}{4^{p} p !} \frac{(s-p) !}{(s-2 p) !} \\
b_{p} & =-\frac{1}{2} \frac{(-1)^{p}}{4^{p} p !} \frac{(s-p-1) !}{(s-2 p-1) !}=-\frac{1}{2} \frac{s-2 p}{s-p} a_{p} .
\end{aligned}
$$

The gauge variation of the Schouten tensor is then indeed a gradient and reads explicitly

$$
\Gamma S=\partial \nu
$$

for a symmetric tensor $\nu$ which is related to $\mu$ as

$$
\nu=\sum_{p=0}^{\lfloor s / 2\rfloor} 2 p a_{p} \delta^{p-1}(\varepsilon \cdot \gamma \cdot) \mu^{[p-1]}-\sum_{p=0}^{\lfloor(s-1) / 2\rfloor} b_{p}(s+1) \gamma_{5} \gamma_{0} \delta^{p} \mu^{[p]} .
$$

The Schouten tensor satisfies the Bianchi identity

$$
U[S] \equiv \partial \cdot S-\not \partial \phi-(s-1) \partial \bar{S}=0,
$$

which is equivalent to the divergencelessness $\partial \cdot G=0$ of the Einstein tensor. Indeed, plugging formula (2.53) into this identity and using the form of the $a_{p}, b_{p}$ coefficients, one gets

$$
U[S]=\frac{1}{s}\left[\sum_{p=0}^{\lfloor s / 2\rfloor} a_{p}(s-2 p) \delta^{p} \partial \cdot G^{[p]}+\sum_{p=0}^{\lfloor(s-1) / 2\rfloor} b_{p}(s-2 p-1) \delta^{p} \gamma \partial \cdot \phi_{T}^{[p]}\right],
$$

which vanishes by virtue of $\partial \cdot G=0$. Similarly, on the parameter $\nu$ for the gauge transformations of the Schouten tensor, the identity equivalent to $\partial \cdot \mu=0$ is

$$
I[\nu] \equiv \partial \cdot \nu+\not \partial \psi+(s-2) \partial \bar{\nu}=0
$$


or, equivalently (using $\gamma^{i} \gamma^{j}=\delta^{i j}+\varepsilon^{i j k} \gamma_{k} \gamma_{5} \gamma_{0}$ ),

$$
\partial \cdot \nu-\frac{1}{2} \gamma_{5} \gamma_{0}(\varepsilon \cdot \partial \cdot \gamma \cdot \nu)+\frac{(s-2)}{2} \partial \bar{\nu}=0 .
$$

These identities are compatible since

$$
U[\partial \nu]=-\frac{(s-1)}{s} \partial I[\nu],
$$

which guarantees (as it should!) that the property $U[S]=0$ is not destroyed by a Weyl transformation.

The Einstein tensor of the Schouten tensor is then the searched-for conformally invariant Cotton tensor

$$
D=(\varepsilon \cdot \partial \cdot)^{s} S .
$$

It is obviously symmetric, divergenceless and invariant under the full gauge and Weyl transformations of the field. It is also gamma-traceless owing to the identity $U[S]=0$; indeed, a short computation shows that

$$
\not D=(\varepsilon \cdot \partial \cdot)^{s-1} U[S]=0 .
$$

As in the spin- $\frac{5}{2}$ case, the proofs of the gauge completeness and the conformal Poincaré lemma heavily rely on the identities (2.60) and (2.62) satisfied by the Schouten tensor and the $\nu$ parameter.

\subsubsection{Gauge completeness}

If $\psi$ is pure gauge, then $D=0$ by construction. To prove the converse, we proceed as before, using the differential $d_{(s)}$. If $D=0$, the Schouten tensor satisfies $d_{(s)}^{s} S=0$, which implies $S=d_{(s)} \nu$ for some rank $s-1$ symmetric tensor $\nu$. Defining $G$ and $\mu$ by inverting the definitions above, this is equivalent to $G=s(\varepsilon \cdot \partial \cdot \gamma \cdot) \mu$. Now, if the ambiguity in $\nu$ allows us to fix $\partial \cdot \mu=0$, implying that $\mu$ is the Einstein tensor of some $\lambda$, we get $G[\psi]=s(\varepsilon \cdot \partial \cdot)^{s}(\gamma \lambda)$, or $(\varepsilon \cdot \partial \cdot)^{s}(\psi-s \gamma \lambda)=0$. Again using the Poincaré lemma for $d_{(s)}$, this implies that $\psi=s(\partial \xi+\gamma \lambda)$ for some $\xi$, showing that $\psi$ is pure gauge.

The key step above is thus again that the ambiguity in $\nu$ allows us to fix $\partial \cdot \mu=0$ or, equivalently, $I[\nu]=0$ (as defined in (2.62)). This can be seen as follows. First of all, the Bianchi identity for the Schouten tensor implies that $I[\nu]$ satisfies $\partial I[\nu]=0$ or, in index notation,

$$
\partial_{\left(i_{1}\right.} I_{\left.i_{2} \cdots i_{s-1}\right)}[\nu]=0 .
$$

The general solution of this equation is [41-43]

$$
I_{i_{1} \cdots i_{s-2}}=\sum_{n=0}^{s-2} p_{i_{1} \cdots i_{s-2} j_{1} \cdots j_{n}}^{(n)} x^{j_{1}} \cdots x^{j_{n}},
$$

where the $p^{(n)}$ are constant tensor with $(s-2, n)$ Young symmetry (in the symmetric convention), i.e.,

$$
\begin{aligned}
p_{i_{1} \cdots i_{s-2} j_{1} \cdots j_{n}}^{(n)} & =p_{\left(i_{1} \cdots i_{s-2}\right) j_{1} \cdots j_{n}}^{(n)}=p_{i_{1} \cdots i_{s-2}\left(j_{1} \cdots j_{n}\right)}^{(n)}, \\
p_{\left(i_{1} \cdots i_{s-2} j_{1}\right) j_{2} \cdots j_{n}}^{(n)} & =0 .
\end{aligned}
$$


On the other hand, the ambiguity in $\nu$ is given by solutions of $\partial \tilde{\nu}=0$, i.e.,

$$
\tilde{\nu}_{i_{1} \cdots i_{s-1}}=\sum_{n=0}^{s-1} q_{i_{1} \cdots i_{s-1} j_{1} \cdots j_{n}}^{(n)} x^{j_{1}} \cdots x^{j_{n}},
$$

where the $q^{(n)}$ are constant tensors with $(s-1, n)$ Young symmetry (again in the symmetric convention). Now, computing $I[\tilde{\nu}]$ shows that we can use this ambiguity to fix $I[\nu]=0$ provided we choose the tensors $q^{(n)}$ such that

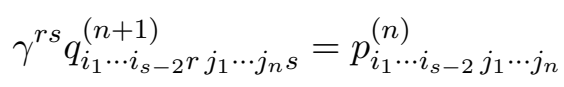

(up to factors that can be absorbed in the $q^{(n)}$ ). That this equation always possesses a solution for arbitrarily given $p$ 's with the $(s-2, n)$ Young symmetry is proven in appendix A.

\subsubsection{Conformal Poincaré lemma}

As was mentioned above, the Cotton tensor is symmetric, divergenceless and gammatraceless. We now want to prove the converse, i.e., that for any symmetric tensor $T$ satisfying $\partial \cdot T=0$ and $T=0$, there exists some $\psi$ such that $T=D[\psi]$. First of all, $\partial \cdot T=0$ implies that there exists a symmetric tensor $S$ of which $T$ is the Einstein tensor,

$$
T=G[S] .
$$

Then, due to $T=0$, this tensor satisfies $(\varepsilon \cdot \partial \cdot)^{s-1} U[S]=0$, which implies

$$
U[S]=\partial \rho
$$

for some symmetric tensor $\rho$ using the appropriate Poincaré lemma. Now, we would like to use the ambiguity in $S$ to cancel $\rho$ so that $U[S]=0$. Indeed, this would imply that $S$ satisfies the Bianchi identity of the Schouten tensor and therefore that some $\psi$ exists such that $S=S[\psi]$, which shows that $T=D[\psi]$.

The ambiguity in $S$ is given by $S \sim S+\partial \nu$ (since it is only defined through its Einstein tensor). Because of equation (2.64), we can fix $U[S]=0$ provided we can solve the differential equation

$$
I[\nu]=\rho
$$

(up to factors and signs that can be absorbed in $\nu$ ). Because of the invertible relation between $\nu$ and $\mu$ (or between $I[\nu]$ and $\partial \cdot \mu$ ), this is equivalent to

$$
\partial \cdot \mu=\hat{\rho},
$$

where $\hat{\rho}$ is an invertible combination of $\rho$ and its traces and gamma-traces. This equation can be solved for $\mu$, which finishes the proof.

\section{Equations of motion as twisted self-duality}

In this section, we rewrite the equations of motion of the fermionic spin $\left(s+\frac{1}{2}\right)$-field in four spacetime dimensions as twisted self-duality conditions on the curvature, supplemented by a purely spatial constraint. 


\subsection{From the Fronsdal to the Riemann tensor}

The equations of motion for the spin $\left(s+\frac{1}{2}\right)$-field $\psi_{\mu_{1} \cdots \mu_{s}}$ are of first-order in derivatives and given by

$$
\mathcal{F}_{\mu_{1} \cdots \mu_{s}}=0
$$

where the Fronsdal tensor is defined as

$$
\mathcal{F}_{\mu_{1} \cdots \mu_{s}}=\not \psi_{\mu_{1} \cdots \mu_{s}}-s \partial_{\left(\mu_{1}\right.} \psi_{\left.\mu_{2} \cdots \mu_{s}\right)}
$$

and the field itself satisfies the trace condition $\psi^{\prime}=0$ (see section 1 for the notation). Under a gauge transformation

$$
\Gamma \psi_{\mu_{1} \cdots \mu_{s}}=s \partial_{\left(\mu_{1}\right.} \xi_{\left.\mu_{2} \cdots \mu_{s}\right)},
$$

the Fronsdal tensor transforms as

$$
\Gamma \mathcal{F}_{\mu_{1} \cdots \mu_{s}}=-s(s-1) \partial_{\left(\mu_{1}\right.} \partial_{\mu_{2}} \xi_{\left.\mu_{3} \cdots \mu_{s}\right)}
$$

or, in index-free notation, $\Gamma \mathcal{F}=d_{(s)}^{2} \xi$, where the differential $d_{(s)}$ satisfies $d_{(s)}^{s+1}=0$. The equations of motion are gauge-invariant if the gauge parameter is gamma-traceless.

The first step is to rewrite these equations in a manner that is invariant under the larger set of traceful gauge transformations; this is done along the lines of $[19,44]$ by going to the (spacetime) Riemann tensor. This formulation contains more derivatives of the field, and the original formulation can then be recovered by fixing the gauge. The Riemann tensor is the $(s, s)$-tensor

$$
R_{\mu_{1} \nu_{1} \cdots \mu_{s} \nu_{s}}=2^{s} \partial_{\left[\nu_{1} \mid\right.} \cdots \partial_{\left[\nu_{s} \mid\right.} \psi_{\left.\left.\mid \mu_{1}\right] \cdots \mid \mu_{s}\right]} .
$$

It is invariant under (3.3) even if the trace parameter is traceful. Taking the gamma-trace of $R$, we get the $(s, s-1)$-tensor

$$
\begin{aligned}
\gamma^{\nu_{1}} R_{\mu_{1} \nu_{1} \cdots \mu_{s} \nu_{s}} & =2^{s} \partial_{\nu_{2}} \cdots \partial_{\nu_{s}}\left(\not \psi_{\mu_{1} \mu_{2} \cdots \mu_{s}}-\partial_{\mu_{1}} \psi_{\mu_{2} \cdots \mu_{s}}\right) \\
& =2^{s} \partial_{\nu_{2}} \cdots \partial_{\nu_{s}}\left(\not \psi_{\mu_{1} \mu_{2} \cdots \mu_{s}}-s \partial_{\left(\mu_{1}\right.} \psi_{\left.\mu_{2} \cdots \mu_{s}\right)}\right) \\
& =2^{s} \partial_{\nu_{2}} \cdots \partial_{\nu_{s}} \mathcal{F}_{\mu_{1} \mu_{2} \cdots \mu_{s}}
\end{aligned}
$$

where the obvious antisymmetrization in $\mu_{k}, \nu_{k}(k \geq 2)$ are not written explicitly to avoid cluttered notation. We have added the necessary terms in the second line (at no cost since partial derivatives commute) to make the Fronsdal tensor appear. In index-free notation, this is

$$
\mathbb{R}=d_{(s)}^{s-1} \mathcal{F} .
$$

Another way to understand why a relation of this type must exist is that, because of the gauge transformation property of the Fronsdal tensor, the quantity $d_{(s)}^{s-1} \mathcal{F}$ is gaugeinvariant and must therefore, like any other local gauge invariant function, be expressible in terms of the Riemann tensor.

Now, $\mathcal{F}=0$ implies $\mathbb{R}=0$; conversely, $\mathbb{R}=0$ implies $\mathcal{F}=d_{(s)}^{2} \zeta$ using the relevant Poincaré lemma, for some $\zeta$ that we can always write as $\zeta=\xi$. This is the equation $\mathcal{F}=0$ up to a traceful gauge transformation. Thus, one can reach $\mathcal{F}=0$ by a gauge transformation when $\mathbb{R}=0$. One can further show [45] that there is enough gauge freedom (when $R=0$ ) to impose also the triple gamma-trace condition on the field $\psi$ itself. 


\subsection{Rewriting as a twisted self-duality condition}

We show in this subsection that the geometrical equation $R=0$ is equivalent to the system

$$
R=-\gamma_{5} \star R, \quad \quad \gamma^{k l} R_{k l} i_{2} j_{2} \cdots i_{s} j_{s}=0,
$$

i.e., the twisted self-duality condition supplemented by a constraint on the purely spatial components. ${ }^{4}$ This is the analog for fermionic fields of the twisted self-duality condition derived in [16] for bosonic fields.

Spin- $\frac{3}{2}$. We first start with the spin- $\frac{3}{2}$ case, which illustrates the main points. The equation $k=0$ is in this case

$$
\gamma^{\mu} R_{\mu \nu}=0, \quad R_{\mu \nu}=\partial_{\nu} \psi_{\mu}-\partial_{\mu} \psi_{\nu}
$$

It is equivalent to the the usual Rarita-Schwinger equation $\gamma^{\mu \nu \rho} R_{\nu \rho}=0$.

- $R=0 \Rightarrow R=-\gamma_{5} \star R$ and $\gamma^{k l} R_{k l i_{2} j_{2} \cdots i_{s} j_{s}}=0$ : first, by contracting with $\gamma^{\nu}$, one sees that $\gamma^{\mu} R_{\mu \nu}=0$ implies $\gamma^{\mu \nu} R_{\mu \nu}=0$ (since $R_{\mu \nu}$ is antisymmetric). Splitting time and space, this is $2 \gamma^{0} \gamma^{i} R_{0 i}+\gamma^{i j} R_{i j}=0$. The first term is the 0 component of $\mathbb{R}=0$ and therefore vanishes; this shows that $R=0$ indeed implies the spatial constraint $\gamma^{i j} R_{i j}=0$.

Then, using the gamma matrix identities

$$
\begin{aligned}
\gamma_{\mu \nu} \gamma^{\rho \sigma} & =\gamma_{\mu \nu}{ }^{\rho \sigma}-4 \delta_{[\mu}^{[\rho} \gamma_{\nu]}^{\sigma]}-2 \delta_{\mu \nu}^{\rho \sigma} \\
\gamma^{\mu \nu \rho} \gamma^{\sigma} & =\gamma^{\mu \nu \rho \sigma}+3 \gamma^{[\mu \nu} \eta^{\rho] \sigma}
\end{aligned}
$$

(valid in all dimensions) and

$$
\gamma_{\mu \nu \rho \sigma}=\varepsilon_{\mu \nu \rho \sigma} \gamma_{5}
$$

with $\varepsilon_{0123}=+1$ (specific to four dimensions), we get

$$
\begin{aligned}
& 0=\gamma_{\mu \nu}{ }^{\rho} \gamma^{\sigma} R_{\rho \sigma}=\varepsilon_{\mu \nu \rho \sigma} \gamma_{5} R^{\rho \sigma}+2 \gamma_{[\mu}{ }^{\sigma} R_{\nu] \sigma} \\
& 0=\gamma_{\mu \nu} \gamma^{\rho \sigma} R_{\rho \sigma}=\varepsilon_{\mu \nu \rho \sigma} \gamma_{5} R^{\rho \sigma}+4 \gamma_{[\mu}{ }^{\sigma} R_{\nu] \sigma}-2 R_{\mu \nu} .
\end{aligned}
$$

Taken together, these two equations imply

$$
R_{\mu \nu}=-\frac{1}{2} \varepsilon_{\mu \nu \rho \sigma} \gamma_{5} R^{\rho \sigma}
$$

which is the twisted self-duality in components.

- $R=-\gamma_{5} \star R$ and $\gamma^{k l} R_{k l i_{2} j_{2} \cdots i_{s} j_{s}}=0 \Rightarrow \mathbb{R}=0$ : splitting space and time, the twisted self-duality is

$$
R_{0 i}=-\frac{1}{2} \varepsilon_{i j k} \gamma_{5} R^{j k}
$$

\footnotetext{
${ }^{4}$ In our conventions $\left(\gamma_{5}\right)^{2}=-1$ which is consistent with $(\star)^{2}=-1$.
} 
Contracting with $\gamma^{i}$ and using the identity $\gamma_{j k}=-\varepsilon_{i j k} \gamma^{i} \gamma_{0} \gamma_{5}$, this gives

$$
2 \gamma^{0} \gamma^{i} R_{0 i}-\gamma^{i j} R_{i j}=0
$$

Using the constraint, this reduces to the zero component of $\mathbb{R}=0$. We are still missing the spatial components of that equation, which read $\gamma^{0} R_{0 i}+\gamma^{k} R_{k i}=0$. This is proved by using the identity

$$
\gamma_{i} \gamma_{j k}=\gamma_{i j k}+2 \delta_{i[j} \gamma_{k]}=-\varepsilon_{i j k} \gamma_{0} \gamma_{5}+2 \delta_{i[j} \gamma_{k]}
$$

which gives, using the constraint and the twisted self-duality condition

$$
\begin{aligned}
0 & =\gamma_{i} \gamma_{j k} R^{j k}=-\varepsilon_{i j k} \gamma_{0} \gamma_{5} R^{j k}+2 \gamma^{k} R_{i k}=2 \gamma_{0} R_{0 i}+2 \gamma^{k} R_{i k} \\
& =-2\left(\gamma^{0} R_{0 i}+\gamma^{k} R_{k i}\right)
\end{aligned}
$$

Arbitrary spin. The proof of the previous section carries over without any change if one adds as many indices as necessary to $R$. This shows the equivalence between $R=0$ and

$$
R=-\gamma_{5} \star R, \quad \gamma^{k l} R_{k l} \mu_{2} \nu_{2} \cdots \mu_{s} \nu_{s}=0,
$$

where the constraint carries additional spacetime indices. To finish the proof in the arbitrary spin case, we therefore need to show that the subset of these constraints with only spatial indices implies all the others components (with one or more zeros).

Using the twisted self-duality condition on other groups of indices, we can dualize every temporal component appearing in the constraint to spatial indices, for example

$$
\gamma^{k l} R_{k l 0 i p_{3} q_{3} \cdots p_{s} q_{s}}=-\frac{1}{2} \varepsilon_{i m n} \gamma^{k l} R_{k l}^{m n}{ }_{p_{3} q_{3} \cdots p_{s} q_{s}}=0 .
$$

This shows that the purely spatial constraint appearing in (3.10) is sufficient.

\subsection{Hamiltonian constraint}

The constraint $\gamma^{k l} R_{k l i_{2} j_{2} \cdots i_{s} j_{s}}=0$ possesses an interesting interpretation in terms of the dynamics: it is equivalent to the constraint that appears in the Hamiltonian formulation, as we now show.

The Fang-Fronsdal action [46] is linear in the first order derivatives and is thus already in Hamiltonian form (up to field redefinitions of the variables). The components $\psi_{0 k_{2} k_{3} \ldots k_{s}}$ with one index equal to 0 are Lagrange multipliers enforcing the "Hamiltonian constraints" on the dynamical variables [18, 47-49]. These constraints arise from the components with one index equal to 0 of the original equations of motion $\mathcal{F}=0$,

$$
0=(s-1) \partial_{\left(k_{2}\right.} \Xi_{\left.k_{3} \ldots k_{s}\right)}+2 \gamma^{l m} \partial_{l} \psi_{m k_{2} \ldots k_{s}}+(s-1) \partial_{\left(k_{2}\right.} \bar{\psi}_{\left.k_{3} \ldots k_{s}\right)},
$$

where

$$
\Xi_{k_{3} \ldots k_{s}}=\psi_{00 k_{3} \ldots k_{s}}-2 \gamma^{0} \gamma^{i} \psi_{0 i k_{3} \ldots k_{s}} .
$$


If we take $s-1$ curls of this expression (i.e., if we compute its Einstein tensor), we will get the vanishing of the gamma-trace of the Einstein tensor of $\psi_{k_{1} \ldots k_{s}}$,

$$
\phi_{r}[\psi]=0 .
$$

Using the spatial $\varepsilon$ tensor, this is equivalent to the constraint written in (3.10) in terms of the Riemann tensor.

\section{Prepotentials}

The fermionic higher-spin conformal geometry provides the tools for introducing prepotentials to write the action for the twisted self-duality equations as a typical, and remarkably simple, prepotential action. It is equivalent to the usual Fronsdal action, where the constraints are solved.

\subsection{Solution of the constraints}

Since the Einstein tensor $G[\psi]$ is symmetric and divergenceless, the conformal Poincaré lemma, proven in section 2.4.2, implies that the constraint $\mathbb{G}_{r}[\psi]=0$ is solved by writing

$$
G[\psi]=D[\chi]
$$

in terms of a prepotential $\chi$, where $D$ is the Cotton tensor. A formula realizing this is simply

$$
\psi[\chi]=S[\chi],
$$

where $S$ is the Schouten tensor, since the Cotton tensor is exactly defined as the Einstein of the Schouten. The simplicity of this formula with respect to the bosonic case seems to be a recurring fact for fermionic fields: see [34, 35], where the same happens for fermionic fields in other dimensions.

Plugging this back into (3.25), we get the equation

$$
0=(s-1) \partial \Xi+(s-1) \partial \bar{S}[\chi]-2(\partial \cdot S[\chi]-\not \partial \phi[\chi])=(s-1) \partial(\Xi-\bar{S}[\chi])
$$

using the identity $\partial \cdot S-\not \partial \$ \$-(s-1) \partial \bar{S}=0$. A particular solution of this equation for $\Xi[\chi]$ is

$$
\Xi[\chi]=\bar{S}[\chi] .
$$

In this way, all the dynamical variables (spatial components of $\psi$ and $\Xi$ ) are expressed in terms of the prepotential $\chi$.

We have chosen a particular solution (4.2) of the equation (4.1). Given the properties of the Einstein tensor, the most general solution will differ from (4.2) by a gauge transformation of $\psi$, and so is physically equivalent to the choice adopted here. Moreover, the relation (4.2) clearly satisfies (by construction of the Schouten tensor) the property that a Weyl transformation of $\chi$ induces a gauge transformation of $\psi$. Finally, we note that the ambiguity in $\Xi$, for fixed $\psi$ 's, is given by a solution of the Killing tensor equation for a tensor with $s-2$ spatial indices. Such a solution is constant or blows up at infinity, and can be dropped if we assume that the spin- $s$ field goes to zero at infinity. 


\subsection{Equations of motion}

A sufficient subset of the twisted self-duality equations is given by the components with at most one zero, i.e.,

$$
R_{0 s j_{2} k_{2} \ldots j_{s} k_{s}}=-\frac{1}{2} \varepsilon_{s p q} \gamma_{5} R_{j_{2} k_{2} \ldots j_{s} k_{s}}^{p q} .
$$

This is proven indirectly below, starting from the action for the field $\psi$. Those equations still contain temporal components of the field; to get rid of those, we take an extra curl, giving the equation

$$
\varepsilon^{i r s} \partial_{r}\left(R_{0 s j_{2} k_{2} \ldots j_{s} k_{s}}+\frac{1}{2} \varepsilon_{s p q} \gamma_{5} R_{j_{2} k_{2} \ldots j_{s} k_{s}}^{p q}\right)=0 .
$$

This equation is equivalent to (4.5): the missing components can be recovered using the appropriate Poincaré lemmas. The fact that only spatial components of $\psi$ appear in this equation can be made more manifest by writing $\varepsilon^{i r s} \partial_{r} R_{0 s j_{2} k_{2} \ldots j_{s} k_{s}}=\frac{1}{2} \varepsilon^{i r s} \dot{R}_{r s j_{2} k_{2} \ldots j_{s} k_{s}}$ using the differential Bianchi identities for the Riemann tensor. Contracting further with epsilon tensors to make the Einstein tensor of $\psi$ appear, we get

$$
\dot{G}^{i_{1} \ldots i_{s}}[\psi]+\gamma_{5} \varepsilon^{i_{1} j k} \partial_{j} G_{k}^{i_{2} \ldots i_{s}}[\psi]=0 .
$$

As emphasized above, this equation (supplemented by the gamma-tracelessness of $G[\psi]$ ) is equivalent to the usual equations of motion for $\psi$. In terms of the prepotential $\chi$, this is

$$
\dot{D}^{i_{1} \ldots i_{s}}[\chi]+\gamma_{5} \varepsilon^{i_{1} j k} \partial_{j} D_{k}^{i_{2} \ldots i_{s}}[\chi]=0 .
$$

We have therefore succeeded in rewriting the equations of motion for the field $\psi$ purely in terms of the prepotential $\chi$; remarkably, they then take the form "sum of time derivative and curl of the Cotton tensor vanishes" that is familiar in the prepotential formulation [13, $16,33-36]$.

\subsection{Action in terms of prepotentials}

The equation above follows from the prepotential action

$$
S[\chi]=-i \int d t d^{3} x \chi_{i_{1} \ldots i_{s}}^{\dagger}\left(\dot{D}^{i_{1} \ldots i_{s}}[\chi]+\gamma_{5} \varepsilon^{i_{1} j k} \partial_{j} D_{k}^{i_{2} \ldots i_{s}}[\chi]\right) .
$$

It is invariant under $\mathrm{SO}(2)$ rotations

$$
\chi \rightarrow e^{\alpha \gamma_{5}} \chi
$$

generated by $\gamma_{5}$, mixing the two chiral components of $\chi$. This extends the results of [17] for spins $1 / 2$ and $3 / 2$ to arbitrary half-integer spin.

In this section, we prove that this is the action that one would obtain starting from the usual Fang-Fronsdal action for $\psi$ and solving the constraints. As in the bosonic case, the argument is indirect and relies on the fact that the action is (almost) uniquely determined by its invariance properties. 
The Fang-Fronsdal action is given by

$$
S=-i \int d^{4} x \bar{\psi}^{\mu_{1} \cdots \mu_{s}} \mathcal{G}_{\mu_{1} \cdots \mu_{s}}
$$

where the tensor $\mathcal{G}$ is given in terms of the Fronsdal tensor (3.2) by

$$
\mathcal{G}_{\mu_{1} \cdots \mu_{s}}=\mathcal{F}_{\mu_{1} \cdots \mu_{s}}-\frac{s}{2} \gamma_{\left(\mu_{1}\right.} \mathcal{F}_{\left.\mu_{2} \cdots \mu_{s}\right)}-\frac{s(s-1)}{4} \eta_{\left(\mu_{1} \mu_{2}\right.} \mathcal{F}_{\left.\mu_{3} \cdots \mu_{s}\right)}^{\prime}
$$

and the field satisfies the triple gamma-trace condition

$$
\psi_{\mu_{4} \cdots \mu_{s}}^{\prime}=0 \text {. }
$$

This trace condition can obviously be solved to express everything in terms of the field variables $\psi_{i_{1} \cdots i_{s}}, \psi_{0 i_{2} \cdots i_{s}}$ and $\psi_{00 i_{3} \cdots i_{s}}$ with at most two temporal indices (see for example [49] for the explicit formulas). Equivalently, one can perform the invertible change of variables (3.26) to eliminate the components with two zeros in favor of $\Xi_{i_{3} \cdots i_{s}}$. Once this is done, the equations of motion show that $\psi_{i_{1} \cdots i_{s}}$ and $\Xi_{i_{3} \cdots i_{s}}$ are dynamical variables, while $\psi_{0 i_{2} \cdots i_{s}}$ is a Lagrange multiplier for the constraint as already indicated above. Therefore, the action (4.11) necessarily takes the form

$$
\begin{aligned}
S=\int d t d^{3} x\left[\Theta^{A}\left(\Psi_{B}\right) \dot{\Psi}_{A}\right. & -\mathcal{H}\left(\Psi_{A}\right) \\
& \left.+\bar{\psi}_{0 i_{2} \cdots i_{s}} \mathcal{C}^{i_{2} \cdots i_{s}}\left(\Psi_{A}\right)+\overline{\mathcal{C}}_{i_{2} \cdots i_{s}}\left(\Psi_{A}\right) \psi^{0 i_{2} \cdots i_{s}}\right] .
\end{aligned}
$$

Here, we wrote $\Psi_{A}=\left(\psi_{i_{1} \cdots i_{s}}, \Xi_{i_{3} \cdots i_{s}}\right)$ for the dynamical variables. Important points are:

1. the variables $\psi_{0 i_{2} \cdots i_{s}}$ only appear as Lagrange multipliers for the constraints $\mathcal{C}\left(\Psi_{A}\right)=$ 0

2. these constraints are equivalent to equation (3.25);

3. since the original action is of first order in derivatives, the functions $\Theta^{A}$ contain no derivatives, while $\mathcal{H}$ contains one spatial derivative only.

The explicit form of $\Theta^{A}, \mathcal{H}$ and $\mathcal{C}$ beyond the features mentioned above are not necessary for the purposes of this argument.

Now, when the constraints are solved in terms of the prepotentials as is the previous section, the Lagrange multipliers $\psi_{0 i_{2} \cdots i_{s}}$ disappear from the action. Then, the kinetic term $\Theta^{A}\left(\Psi_{B}\right) \dot{\Psi}_{A}$ must be a function of the prepotentials with one time derivative and $2 s$ spatial derivatives (since the fields $\Psi_{A}$ are expressed as $s$ spatial derivatives of the prepotential $\chi$ ). Since it must be invariant under gauge and Weyl transformations of the prepotential, it must (up to integration by parts) take the form of the first term of the action (4.9). Similarly, the Hamiltonian density must contain $2 s+1$ derivatives and, by the same invariance argument, must take the form of the second term of (4.9). The relative factor of these two terms is fixed by the fact that this action should yield (4.8) as equations of motion with the relative factor written there, since these equations are a consequence of the original Fronsdal equations as we saw in the previous sections. 


\section{Comments and conclusions}

In this paper, we have developed the (linearized) conformal geometry of higher-spin fermionic fields in three dimensions. The difficulty comes from the fact that the Weyl tensor identically vanishes in three dimensions so that the conformal invariants must be constructed out of the "Cotton tensor", which generalizes the tensor with the same name of gravity and involves higher order derivatives. This tensor was defined and its central properties (gauge completeness and conformal Poincaré lemma) were established.

We then used these conformal tools to introduce the "prepotentials", which provide the explicit solution of the constraint equations resulting from the Fang-Fronsdal action [46]. The reformulation in terms of the prepotentials, intimately connected with the twisted self-duality reformulation (see eq. (4.8)), puts on the same footing the fermionic fields and the bosonic fields, for which a similar prepotential formulation was achieved in $[15,16]$ starting from the Fronsdal action [50].

The prepotential formulation possesses two striking features:

- The prepotential action enjoys both generalized diffeomorphism invariance (like the higher-spin (Fang-)Fronsdal action) and generalized Weyl invariance. This is true for all spins and holds both in the bosonic and fermionic cases. Furthermore, the spatial dimension is the critical dimension where Weyl geometry requires the introduction of the Cotton tensor since the Weyl tensor identically vanishes. This is also a feature that appears to be universal and was found to hold in higher dimensions where the prepotentials have a non trivial Young mixed symmetry [32-36]. The emergence of higher-spin Weyl invariance deserves further understanding.

- The resulting prepotential action always takes the same simple form, for all spins, namely "prepotentials $\times$ (time derivative of the Cotton tensors + curl of the Cotton tensors)" (see also table 1 at the end of this work). This is suggestive that the sum over all spins of the actions should enjoy remarkable symmetry properties, in particular $s p(8)$-symmetry $[51,52]$ or hypersymmetry [47, 53-56] (for more recent work see $[18,57,58])$.

Finally, it would be of interest to extend the analysis of this paper to (anti-)de Sitter backgrounds, following the spin-2 case $[14,59,60]$. In that context, we point out reference [61], which gives in its Appendix $\mathrm{C}$ a construction of higher-spin Cotton tensors for generic conformally flat space in three dimensions. That interesting construction proceeds along different lines from those followed in our paper and starts from the Fang-Fronsdal field strengths.

\section{Acknowledgments}

We thank Xavier Bekaert, Andrea Campoleoni and Sergio Hörtner for useful discussions. V.L. and A.L. are Research Fellows at the Belgian F.R.S.-FNRS. This work was partially supported by the ERC through the "High-Spin-Grav" Advanced Grant and by FNRSBelgium (convention FRFC PDR T.1025.14 and convention IISN 4.4503.15). This project 
has received funding from the European Research Council (ERC) under the European Union's Horizon 2020 research and innovation programme ("Exceptional Quantum Gravity", grant agreement No 740209).

\section{A Proof of $\gamma q=p$}

We want to prove that equation (2.72) of section 2.4.1

$$
\gamma^{r s} q_{i_{1} \cdots i_{s-2} r j_{1} \cdots j_{n} s}=p_{i_{1} \cdots i_{s-2} j_{1} \cdots j_{n}}
$$

always possesses a solution for arbitrarily given $p$ 's with $(s-2, n)$ Young symmetry (the $q$ 's have $(s-1, n+1)$ Young symmetry). It is a linear system of inhomogeneous equations

$$
A_{\alpha}{ }^{A} q_{A}=p_{\alpha}
$$

that has a solution for given $p_{\alpha}$ if and only if the given $p_{\alpha}$ fulfills $y^{\alpha} p_{\alpha}=0$ for any left eigenvector $y^{\alpha}$ of the matrix $A_{\alpha}{ }^{A}$ with eigenvalue zero (i.e., $y^{\alpha} A_{\alpha}{ }^{A}=0$ ). Since we want no restriction on $p_{\alpha}$, the matrix $A_{\alpha}{ }^{A}$ should have no left eigenvector for the eigenvalue zero. That is, the system $A_{\alpha}{ }^{A} q_{A}=p_{\alpha}$ has always a solution for arbitrary $p$ 's if and only if the only solution to the equations $y^{\alpha} A_{\alpha}{ }^{A}=0$ is $y^{\alpha}=0$. In our case (A.2) explicitly corresponds to

$$
A_{i_{1} \cdots i_{s-2} j_{1} \cdots j_{n}}{ }^{i_{1}^{\prime} \cdots i_{s-2}^{\prime} r^{\prime} j_{1}^{\prime} \cdots j_{n}^{\prime} s^{\prime}} q_{i_{1}^{\prime} \cdots i_{s-2}^{\prime} r^{\prime} j_{1}^{\prime} \cdots j_{n}^{\prime} s^{\prime}}=p_{i_{1} \cdots i_{s-2} j_{1} \cdots j_{n}}
$$

where

$$
A_{i_{1} \cdots i_{s-2} j_{1} \cdots j_{n}}{ }^{i_{1}^{\prime} \cdots i_{s-2}^{\prime}} r^{\prime} j_{1}^{\prime} \cdots j_{n}^{\prime} s^{\prime}=\mathbb{P}\left(\delta_{i_{1}}^{i_{1}^{\prime}} \cdots \delta_{i_{s-2}}^{i_{s-2}^{\prime}} \gamma^{r^{\prime} s^{\prime}} \delta_{j_{1}}^{j_{1}^{\prime}} \cdots \delta_{j_{n}}^{j_{n}^{\prime}}\right)
$$

and $\mathbb{P}$ projects on the appropriate Young symmetries, i.e., $(s-2, n)$ for the lower indices and $(s-1, n+1)$ for the higher indices. Transposing spinor indices, this is equivalent to showing that the only solution to the equation

$$
\gamma_{(r \mid(s} y_{\left.\left.i_{1} \cdots i_{s-2}\right) \mid j_{1} j_{2} \cdots j_{n}\right)}=0
$$

with $y_{i_{1} \cdots i_{s-2}} j_{1} j_{2} \cdots j_{n}$ of $p$-symmetry type $(s-2, n)$ is $y_{i_{1} \cdots i_{s-2}} j_{1} j_{2} \cdots j_{n}=0$.

As a first step we set $i_{k}=s$ and $j_{k}=r$ which leads for $r \neq s$, for which $\gamma_{r s}$ is invertible, to $\gamma_{r s} y_{s \cdots s r \cdots r}=0$ which implies

$$
y_{s \cdots s r \cdots r}=0 .
$$

In this section we do no sum over the $s$ and $r$ indices. We now use (A.6) to "free" one of the indices of the first group so that $\gamma_{(r \mid(s} y_{\left.\left.i_{1} s \cdots s\right) \mid r \cdots r\right)}=\gamma_{r(s} y_{\left.i_{1} s \cdots s\right) r \cdots r}=\# \gamma_{r s} y_{i_{1} s \cdots s r \cdots r}=0$, which leads for $r \neq s$ to $\gamma_{r s} y_{i_{1} s \cdots s r \cdots r}=0$ and therefore

$$
y_{i_{1} s \cdots s r \cdots r}=0
$$




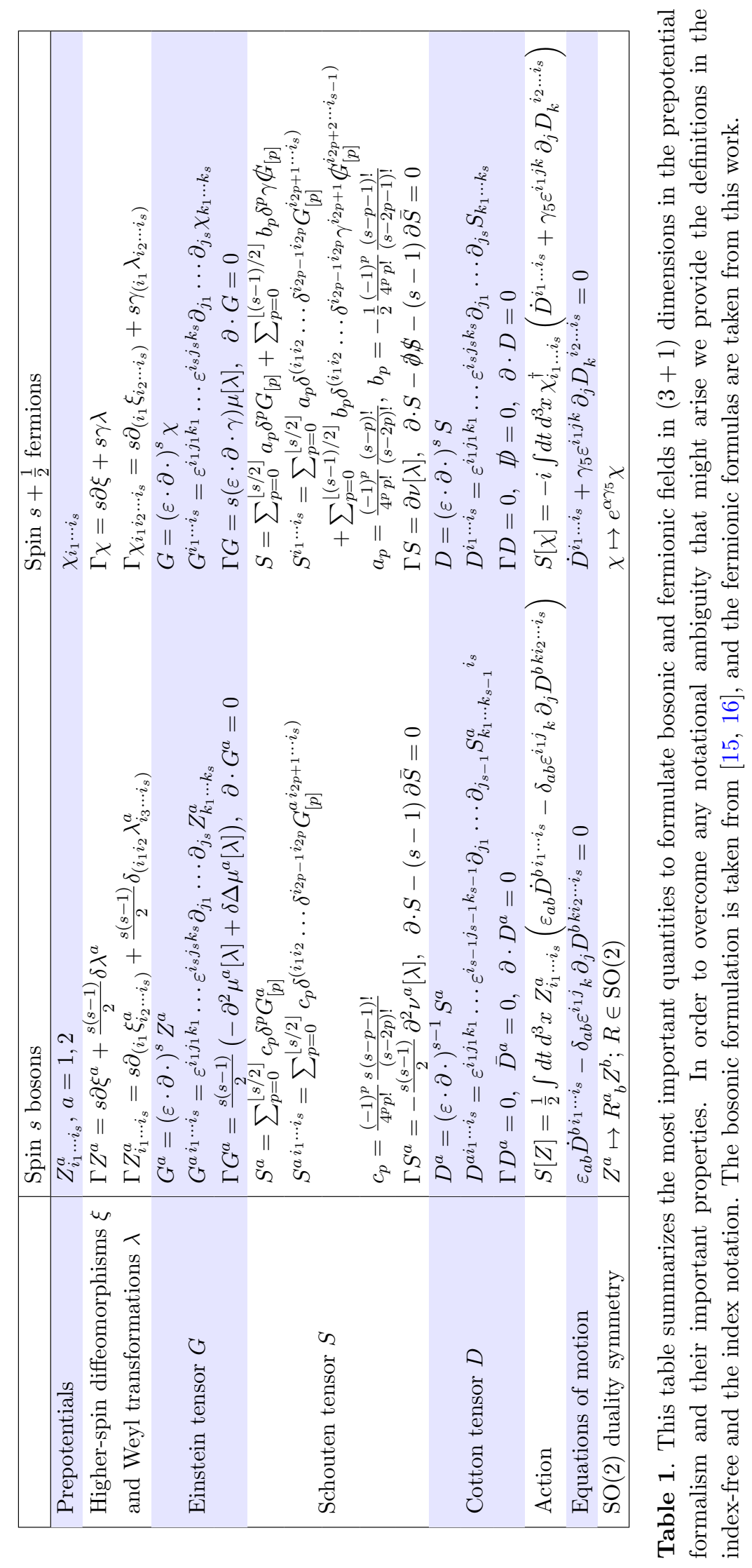


We denote with \# strictly positive constants whose values are not relevant for the proof. Repeating this argument for $\gamma_{(r \mid(s} y_{\left.\left.i_{1} i_{2} s \cdots s\right) \mid r \cdots r\right)}=0$ implies that $y_{i_{1} i_{2} s \cdots s r \cdots r}=0$. We can reiterate on the first group of indices and repeat the same analysis for the second group to get

$$
y_{i_{1} \cdots i_{s-2} r \cdots r}=0=y_{s \cdots s j_{1} \cdots j_{n}} .
$$

We next use these relations to connect the two indices groups since $\gamma_{(r \mid(s} y_{\left.\left.i_{1} s \cdots s\right) \mid j_{1} r \cdots r\right)}=$ $\# \gamma_{r s} y_{i_{1} s \cdots s j_{1} r \cdots r}=0$ which leads for $r \neq s$ to $y_{i_{1} s \cdots s j_{1} r \cdots r}=0$. Now we can systematically "free" the indices, e.g., $\gamma_{(r \mid(s} y_{\left.\left.i_{1} i_{2} s \cdots s\right) \mid j_{1} r \cdots r\right)}=0$ implies $y_{i_{1} i_{2} s \cdots s j_{1} r \cdots r}=0$. Repeating this analysis iteratively on both groups of indices shows that the only solution of (A.5) is $y_{i_{1} \cdots i_{s-2}} j_{1} j_{2} \cdots j_{n}=0$ which completes the proof.

Open Access. This article is distributed under the terms of the Creative Commons Attribution License (CC-BY 4.0), which permits any use, distribution and reproduction in any medium, provided the original author(s) and source are credited.

\section{References}

[1] E.S. Fradkin and A.A. Tseytlin, Conformal supergravity, Phys. Rept. 119 (1985) 233 [INSPIRE].

[2] C.N. Pope and P.K. Townsend, Conformal higher spin in $(2+1)$-dimensions, Phys. Lett. B 225 (1989) 245 [INSPIRE].

[3] E.S. Fradkin and V.Ya. Linetsky, Cubic interaction in conformal theory of integer higher spin fields in four-dimensional space-time, Phys. Lett. B 231 (1989) 97 [INSPIRE].

[4] E.S. Fradkin and V.Ya. Linetsky, A superconformal theory of massless higher spin fields in $D=(2+1)$, Mod. Phys. Lett. A 4 (1989) 731 [INSPIRE].

[5] M.A. Vasiliev, Conformal higher spin symmetries of $4 D$ massless supermultiplets and $\operatorname{OSp}(L, 2 M)$ invariant equations in generalized (super)space, Phys. Rev. D 66 (2002) 066006 [hep-th/0106149] [INSPIRE].

[6] A.Y. Segal, Conformal higher spin theory, Nucl. Phys. B 664 (2003) 59 [hep-th/0207212] [INSPIRE].

[7] O.V. Shaynkman, I.Yu. Tipunin and M.A. Vasiliev, Unfolded form of conformal equations in $M$ dimensions and $\mathrm{O}(M+2)$ modules, Rev. Math. Phys. 18 (2006) 823 [hep-th/0401086] [INSPIRE].

[8] M.A. Vasiliev, On conformal, $\mathrm{SL}(4, \mathbb{R})$ and $\mathrm{Sp}(8, R)$ symmetries of $4 d$ massless fields, Nucl. Phys. B 793 (2008) 469 [arXiv:0707.1085] [INSPIRE].

[9] R.R. Metsaev, Ordinary-derivative formulation of conformal totally symmetric arbitrary spin bosonic fields, JHEP 06 (2012) 062 [arXiv:0709.4392] [INSPIRE].

[10] R.R. Metsaev, Gauge invariant two-point vertices of shadow fields, AdS/CFT and conformal fields, Phys. Rev. D 81 (2010) 106002 [arXiv:0907.4678] [INSPIRE].

[11] S. Deser and C. Teitelboim, Duality transformations of abelian and nonabelian gauge fields, Phys. Rev. D 13 (1976) 1592 [INSPIRE].

[12] M. Henneaux and C. Teitelboim, Duality in linearized gravity, Phys. Rev. D 71 (2005) 024018 [gr-qc/0408101] [INSPIRE]. 
[13] C. Bunster, M. Henneaux and S. Hortner, Gravitational electric-magnetic duality, gauge invariance and twisted self-duality, J. Phys. A 46 (2013) 214016 [Erratum ibid. A 46 (2013) 269501] [arXiv: 1207.1840] [INSPIRE].

[14] B. Julia, J. Levie and S. Ray, Gravitational duality near de Sitter space, JHEP 11 (2005) 025 [hep-th/0507262] [INSPIRE].

[15] M. Henneaux, S. Hörtner and A. Leonard, Higher spin conformal geometry in three dimensions and prepotentials for higher spin gauge fields, JHEP 01 (2016) 073 [arXiv: 1511.07389] [INSPIRE].

[16] M. Henneaux, S. Hörtner and A. Leonard, Twisted self-duality for higher spin gauge fields and prepotentials, Phys. Rev. D 94 (2016) 105027 [Erratum ibid. D 97 (2018) 049901] [arXiv: 1609.04461] [INSPIRE].

[17] C. Bunster and M. Henneaux, Supersymmetric electric-magnetic duality as a manifest symmetry of the action for super-Maxwell theory and linearized supergravity, Phys. Rev. D 86 (2012) 065018 [arXiv:1207.1761] [INSPIRE].

[18] C. Bunster, M. Henneaux, S. Hörtner and A. Leonard, Supersymmetric electric-magnetic duality of hypergravity, Phys. Rev. D 90 (2014) 045029 [Erratum ibid. D 95 (2017) 069908] [arXiv: 1406.3952] [INSPIRE].

[19] T. Damour and S. Deser, 'Geometry' of spin 3 gauge theories, Ann. Inst. H. Poincare Phys. Theor. 47 (1987) 277.

[20] T. Basile, R. Bonezzi and N. Boulanger, The Schouten tensor as a connection in the unfolding of $3 D$ conformal higher-spin fields, JHEP 04 (2017) 054 [arXiv:1701.08645] [INSPIRE].

[21] E.A. Bergshoeff, O. Hohm and P.K. Townsend, On higher derivatives in $3 D$ gravity and higher spin gauge theories, Annals Phys. 325 (2010) 1118 [arXiv:0911.3061] [INSPIRE].

[22] E.A. Bergshoeff et al., A spin-4 analog of 3D massive gravity, Class. Quant. Grav. 28 (2011) 245007 [arXiv: 1109.0382] [INSPIRE].

[23] B.E.W. Nilsson, Towards an exact frame formulation of conformal higher spins in three dimensions, JHEP 09 (2015) 078 [arXiv:1312.5883] [INSPIRE].

[24] B.E.W. Nilsson, On the conformal higher spin unfolded equation for a three-dimensional self-interacting scalar field, JHEP 08 (2016) 142 [arXiv: 1506.03328] [INSPIRE].

[25] H. Linander and B.E.W. Nilsson, The non-linear coupled spin 2-spin 3 Cotton equation in three dimensions, JHEP 07 (2016) 024 [arXiv: 1602.01682] [INSPIRE].

[26] R. Andringa et al., Massive 3D supergravity, Class. Quant. Grav. 27 (2010) 025010 [arXiv: 0907.4658] [INSPIRE].

[27] S.M. Kuzenko and D.X. Ogburn, Off-shell higher spin $N=2$ supermultiplets in three dimensions, Phys. Rev. D 94 (2016) 106010 [arXiv:1603.04668] [InSPIRE].

[28] S.M. Kuzenko, Higher spin super-Cotton tensors and generalisations of the linear-chiral duality in three dimensions, Phys. Lett. B 763 (2016) 308 [arXiv:1606.08624] [INSPIRE].

[29] S.M. Kuzenko and M. Tsulaia, Off-shell massive $N=1$ supermultiplets in three dimensions, Nucl. Phys. B 914 (2017) 160 [arXiv:1609.06910] [INSPIRE].

[30] M. Dubois-Violette and M. Henneaux, Generalized cohomology for irreducible tensor fields of mixed Young symmetry type, Lett. Math. Phys. 49 (1999) 245 [math/9907135] [INSPIRE]. 
[31] M. Dubois-Violette and M. Henneaux, Tensor fields of mixed Young symmetry type and $N$ complexes, Commun. Math. Phys. 226 (2002) 393 [math/0110088] [INSPIRE].

[32] C. Bunster, M. Henneaux and S. Hörtner, Twisted self-duality for linearized gravity in D dimensions, Phys. Rev. D 88 (2013) 064032 [arXiv:1306.1092] [InSPIRE].

[33] M. Henneaux, V. Lekeu and A. Leonard, Chiral tensors of mixed Young symmetry, Phys. Rev. D 95 (2017) 084040 [arXiv:1612.02772] [InSPIRE].

[34] M. Henneaux, V. Lekeu and A. Leonard, The action of the (free) (4,0)-theory, JHEP 01 (2018) 114 [Erratum ibid. 05 (2018) 105] [arXiv:1711.07448] [INSPIRE].

[35] V. Lekeu and A. Leonard, Prepotentials for linearized supergravity, arXiv:1804.06729 [INSPIRE].

[36] M. Henneaux, V. Lekeu, J. Matulich and S. Prohazka, The action of the (free) $\mathcal{N}=(3,1)$ theory in six spacetime dimensions, JHEP 06 (2018) 057 [arXiv:1804.10125] [INSPIRE].

[37] S. Deser, J.H. Kay and K.S. Stelle, Hamiltonian formulation of supergravity, Phys. Rev. D 16 (1977) 2448 [INSPIRE].

[38] S. Deser and D. Seminara, Duality invariance of all free bosonic and fermionic gauge fields, Phys. Lett. B 607 (2005) 317 [hep-th/0411169] [INSPIRE].

[39] S. Deser and D. Seminara, Duality invariance of $s \geq \frac{3}{2}$ fermions in AdS, Phys. Lett. B 738 (2014) 323 [arXiv:1409.3545] [INSPIRE].

[40] B. de Wit and D.Z. Freedman, Systematics of higher spin gauge fields, Phys. Rev. D 21 (1980) 358 [INSPIRE].

[41] G. Thompson, Killing tensors in spaces of constant curvature, J. Math. Phys. 27 (1986) 2693.

[42] T. Wolf, Structural equations for Killing tensors of arbitrary rank, Comp. Phys. Commun. 115 (1998) 316.

[43] R.G. McLenaghan, M. Robert and R.G. Smirnov, Killing tensors as irreducible representations of the general linear group, C. R. Acad. Sci. Paris I 339 (2004) 621.

[44] X. Bekaert and N. Boulanger, On geometric equations and duality for free higher spins, Phys. Lett. B 561 (2003) 183 [hep-th/0301243] [INSPIRE].

[45] D. Francia and A. Sagnotti, On the geometry of higher spin gauge fields, Class. Quant. Grav. 20 (2003) S473 [hep-th/0212185] [INSPIRE].

[46] J. Fang and C. Fronsdal, Massless fields with half integral spin, Phys. Rev. D 18 (1978) 3630 [INSPIRE].

[47] C. Aragone and S. Deser, Hamiltonian form for massless higher spin fermions, Phys. Rev. D 21 (1980) 352 [inSPIRE].

[48] A. Borde, Hamiltonian formalism for the spin 5/2 gauge field, Phys. Rev. D 26 (1982) 407 [INSPIRE].

[49] A. Campoleoni, M. Henneaux, S. Hörtner and A. Leonard, Higher-spin charges in Hamiltonian form. II. Fermi fields, JHEP 02 (2017) 058 [arXiv:1701.05526] [INSPIRE].

[50] C. Fronsdal, Massless fields with integer spin, Phys. Rev. D 18 (1978) 3624 [INSPIRE].

[51] C. Fronsdal, Massless particles, orthosymplectic symmetry and another type of Kaluza-Klein theory, Math. Phys. Stud. 8 (1986) 163. 
[52] O.A. Gelfond and M.A. Vasiliev, Symmetries of higher-spin current interactions in four dimensions, Theor. Math. Phys. 187 (2016) 797 [arXiv:1510.03488] [INSPIRE].

[53] F.A. Berends, J.W. van Holten, P. van Nieuwenhuizen and B. de Wit, On Spin 5/2 gauge fields, Phys. Lett. B 83 (1979) 188 [Erratum ibid. B 84 (1979) 529].

[54] F.A. Berends, J.W. van Holten, B. de Wit and P. van Nieuwenhuizen, On spin $5 / 2$ gauge fields, J. Phys. A 13 (1980) 1643 [InSPIRE].

[55] C. Aragone and S. Deser, Higher spin vierbein gauge fermions and hypergravities, Nucl. Phys. B 170 (1980) 329 [INSPIRE].

[56] C. Aragone and S. Deser, Hypersymmetry in $D=3$ of coupled gravity massless spin $5 / 2$ system, Class. Quant. Grav. 1 (1984) L9 [InSPIRE].

[57] O. Fuentealba, J. Matulich and R. Troncoso, Extension of the Poincaré group with half-integer spin generators: hypergravity and beyond, JHEP 09 (2015) 003 [arXiv: 1505.06173] [INSPIRE].

[58] O. Fuentealba, J. Matulich and R. Troncoso, Asymptotically flat structure of hypergravity in three spacetime dimensions, JHEP 10 (2015) 009 [arXiv: 1508.04663] [INSPIRE].

[59] N. Boulanger, A. Campoleoni and I. Cortese, Dual actions for massless, partially-massless and massive gravitons in (A)dS, Phys. Lett. B 782 (2018) 285 [arXiv:1804.05588] [INSPIRE].

[60] N. Boulanger, A. Campoleoni, I. Cortese and L. Traina, Spin-2 twisted duality in (A)dS, arXiv: 1807.04524 [INSPIRE].

[61] S.M. Kuzenko and M. Ponds, Topologically massive higher spin gauge theories, JHEP 10 (2018) 160 [arXiv : 1806. 06643] [INSPIRE]. 